\title{
Introduction of Electric Vehicle Charging Stations to University Campuses: A Case Study for the University of Georgia from 2014 to 2017
}

\author{
Sierra Hovet ${ }^{1}$, Blair Farley ${ }^{2}$, Jason Perry ${ }^{3}$, Kevin Kirsche ${ }^{3}$, Michael Jerue ${ }^{4}$ \\ and Zion Tsz Ho Tse ${ }^{5, *}$ (i) \\ 1 College of Engineering, University of Georgia, Athens, GA 30602, USA; sierrahovet@gmail.com \\ 2 Southern Company Services, Inc., 42 Inverness Center Pl, Birmingham, AL 35242, USA; \\ kbfarley@southernco.com \\ 3 Office of Sustainability, University of Georgia, Athens, GA 30602, USA; jcperry@uga.edu (J.P.); \\ kkirsche@uga.edu (K.K.) \\ 4 JuiceCar LLC, 755 W Vincent Dr, Athens, GA 30607, USA; mike.jerue@gmail.com \\ 5 School of Electrical and Computer Engineering, University of Georgia, Athens, GA 30602, USA \\ * Correspondence: ziontse@uga.edu; Tel.: +1-706-542-4189
}

Received: 28 March 2018; Accepted: 25 May 2018; Published: 7 June 2018

\begin{abstract}
Electric vehicles (EVs) are becoming increasingly popular in the United States of America (USA). EVs attract buyers with benefits including energy efficiency and environmental friendliness. As EV usage grows, more public spaces are installing EV charging stations. This paper presents a comprehensive analysis of EV charging station usage at the University of Georgia (UGA) in Athens, Georgia. Three ChargePoint EV charging stations at UGA were used to collect data about each of 3204 charging events that occurred from 10 April 2014 to 20 June 2017. The charging event data included start date, start time, length of parking time, length of charging time, amount of energy delivered, and the postal code entered by the user during ChargePoint account registration. Analytical methods were proposed to obtain information about EV charging behavior, charging station occupancy, and geolocation of charging station users. The methodology presented here was time- and cost-effective, as well as scalable to other organizations that own charging stations. Because this study took place at a university, the results presented here can be used as a reference for EV charging station usage in other college towns in the USA that do not have EV charging stations but are planning to develop EV infrastructure.
\end{abstract}

Keywords: electric vehicle; vehicle usage analysis; electric vehicle charging station

\section{Introduction}

Electric vehicles (EV) are becoming increasingly popular in the United States of America (USA). From 2011 to 2017, yearly sales of all-electric vehicles and plug-in hybrids rose from $0.14 \%$ to $1.09 \%$ of total vehicle sales in the USA [1]. EV adoption in the USA is driven in part by inherent benefits of EVs, such as energy efficiency and environmental friendliness [2]. It is also spurred by government promotion and financial incentives. Purchasing a new EV may qualify owners to receive a federal tax credit [2-4] as well as state, city, or utility incentives depending on location [4]. Such incentives have been offered as part of an effort to decrease the USA's reliance on oil and diversify fuel sources within the transportation sector [5], which is especially important as $92 \%$ of fuel used for transportation is petroleum-based [6]. Increasing EV use is one way to increase alternative domestic fuel use, which may contribute to more reliable energy supply, stable prices, and reduced greenhouse gas emissions [5]. 
Electric vehicles require specialized EV charging stations [7] and driving range may be a source of concern when considering an EV. However, plug-in hybrids can alleviate range anxiety by allowing the driver to rely on gasoline when the battery is depleted. The 2018 Chevrolet Volt, for instance, can travel 53 miles with just the battery and up to 420 miles with both a full charge and a full tank of gas [8]. Additionally, although some all-electric EVs have limited range [3], advancements in newer models are reflected in extended driving ranges [9]. For example, with a single charge, the 2018 Chevrolet Bolt EV has a range of 238 miles [10], the Tesla Model 3 has a range of 220 miles [11], and the Tesla Model S has a range of 335 miles [12]. As technology improves, future all-electric EVs can be expected to be competitive with standard vehicles in terms of both range and cost. Installing public EV charging stations in optimal locations is key to increasing driving range [13-15] thereby allowing and encouraging EV use in the surrounding area [14-16].

Many studies have been conducted on possible methods to assist with selecting the best locations for EV charging stations. Kameda and Mukai aimed to identify the optimal locations of charging stations for an on-demand bus system serviced by all-electric buses [13]. Chen et al. created an algorithm to determine optimal locations of charging stations based on parking information as well as many other regional statistics. The selection process aimed to minimize station access costs and penalize unmet demand [14]. Ge et al. proposed a planning method based on zoning the planning area by the grid partition method, optimizing the charging station location in each zone based on traffic density and charging capacity, and iteratively adjusting and recalculating charging station locations [15]. Frade et al. used a maximal covering model to choose the number of charging stations needed and the best locations for the stations to meet as much demand as possible [16]. Oda et al. used charging records to estimate future waiting times for charging and conducted a cost-benefit analysis for installing additional charging units [17]. Aziz et al. developed a system to increase the charging rate of EV chargers while maintaining the rated power capacity [18]. Azadfar et al. reviewed the main technical, environmental, and economical factors that can affect EV driver charging behavior and overall charging patterns [19]. Moon et al. analyzed the consumer demand for EV charging and found that most charging occurs in the evening. During the day, public chargers are used more often than private chargers, and faster public chargers are typically used during peak load times despite the higher cost [20].

However, there is a lack of research focusing on the introduction of EV charging stations to university campuses. Universities are unique locations to promote EV adoption for the next generation. Many universities are also interested in sustainability and environmentally friendly practices. When universities and industry meet to discuss possible partnerships and investments, there is likely to be a lack of either relevant data or methodology to organize relevant data so that it is useful for infrastructure planning. The aim of this study was to act as reference that can be used in two different ways: (1) if a university does not have any existing charging station infrastructure, this case study can be used as a rough guide for potential expectations of charging station usage on campus; and (2) if a university already has charging stations, this case study can be used to guide a simple analysis of charging station usage that will be practical for decision-making.

This study proposes a methodology to analyze EV charging station usage on a college campus. The University of Georgia (UGA) in Athens, Georgia was used as a case study to show how to apply the methodology. UGA has a population of around 35,000, and Athens has a total population of around 120,000 [21]. There are around 1000 college towns in the USA, and at least 50 have a comparable population to UGA [22,23]. There are currently three ChargePoint charging stations available on UGA campus [24]. This case study used data collected directly from the charging stations on campus to provide information about EV charging behavior, charging station occupancy, and geolocation of charging station users on the UGA campus from 10 April 2014 to 20 June 2017. The results are presented in Section 2. The results, limitations, and future work are discussed in Section 3. Section 4 describes the methods and materials, and the conclusions are listed in Section 5.

The data collection method is time- and cost-effective because ChargePoint charging stations collect data about every charging event, and these data can be quickly obtained free of charge by the charging 
station owners. The methods used in this study are reproducible for other cities, and the results will be useful for decision making about EV charging infrastructure development on college campuses and the surrounding community. The methodology will be especially useful for other college towns that plan to build additional charging stations or introduce EV charging infrastructure to their campus. If installing additional charging stations, the methodology can be used to examine past charging behavior at existing charging stations. If a college campus does not have existing charging stations, this case study can be used as a reference for possible expectations of charging behavior, occupancy, and driver locations.

\section{Results}

\subsection{Charging Behavior}

From the histogram of charging time (Figure 1), it can be seen that the distribution of charging time was skewed to the left. The most common length of charging time was in the range of $(1,2] \mathrm{h}$. Overall, $77.4 \%$ of charging events had a charging time of $3 \mathrm{~h}$ or less.

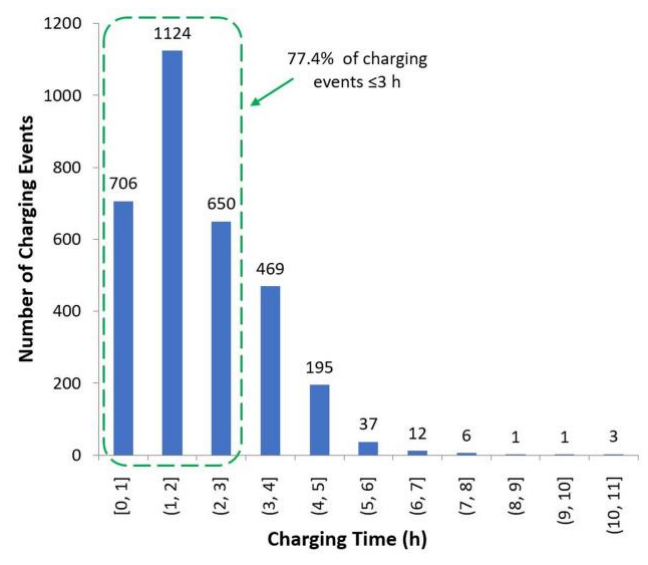

Figure 1. Histogram of the charging time in each charging event.

EV users may remain parked at the charging station after charging is complete. Non-charging parking time is defined as the total parking time minus the charging time. Figure 2 is the histogram of non-charging parking time. Similar to charging time, the distribution of non-charging parking time was also skewed to the left. By far the most common length of non-charging parking time was in the range $[0,1] \mathrm{h}$, with $90.3 \%$ of charging events in this category. Additionally, the graph can be used to calculate the number of charging events within any desired range. For example, $99.2 \%$ of charging events had $[0,5] \mathrm{h}$ of non-charging parking time.

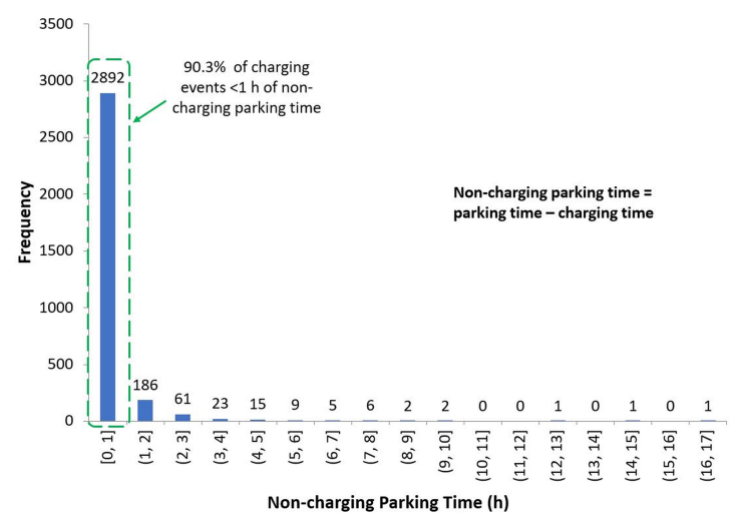

Figure 2. Histogram of the non-charging parking time in each charging event. 
The amount of energy delivered during each EV charging event is shown in Figure 3. Energy delivery per charging event fluctuated over time but did not follow any discernible trend. It was calculated that $91.8 \%$ of charges were below $18 \mathrm{kWh}$ per charge.

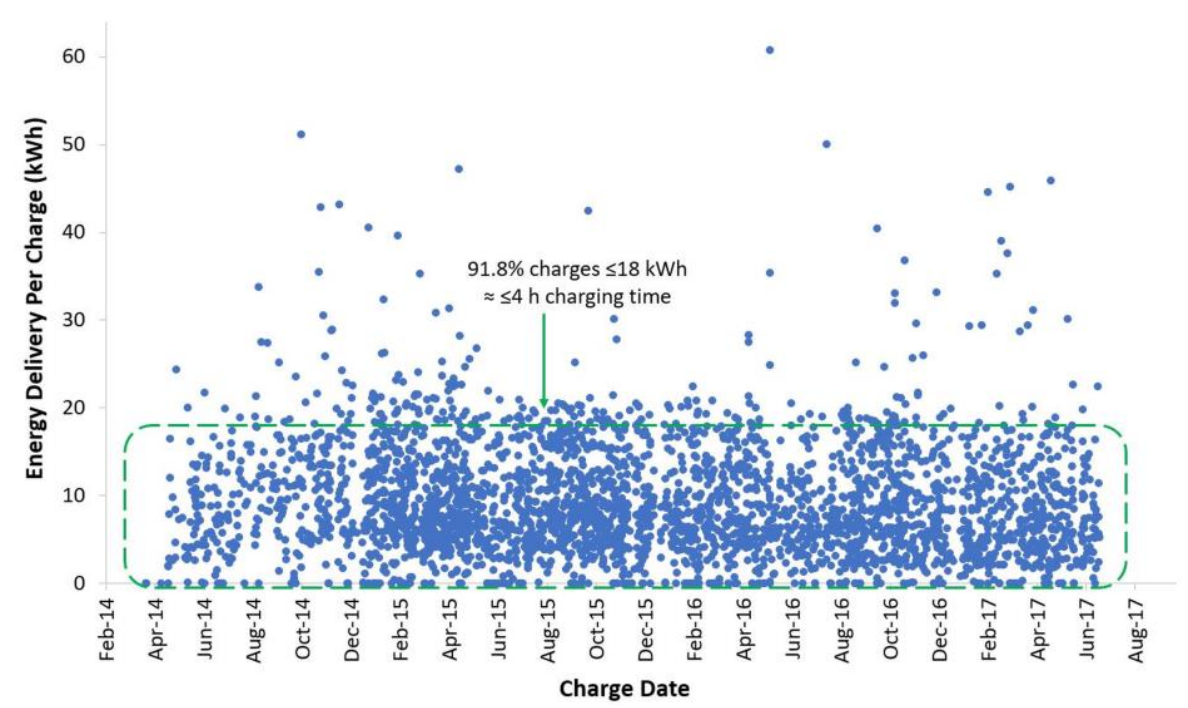

Figure 3. Scatterplot of energy delivery for each charging event.

In Figure 4, the relationship between energy delivery and charging time for each charging event can be seen. Two straight lines show the power region that the ChargePoint chargers provide, ranging from approximately $3 \mathrm{~kW}$ to $6 \mathrm{~kW}$. The average actual power per charging event was calculated to be $4.4 \mathrm{~kW}$ with a standard deviation of $1.57 \mathrm{~kW}$. Since the average power delivery at the charging stations was $4.4 \mathrm{~kW}$, transferring $18 \mathrm{kWh}$ of energy would take approximately $4.1 \mathrm{~h}$; this information was added to Figure 3. It was estimated that approximately $91.8 \%$ of charging events would last less than or equal to $4.1 \mathrm{~h}$ because $91.8 \%$ of charging events were below $18 \mathrm{kWh}$. By analyzing the raw data, it was found that $93.2 \%$ of charging events lasted less than or equal to $4.1 \mathrm{~h}$, so the estimate was reasonable.

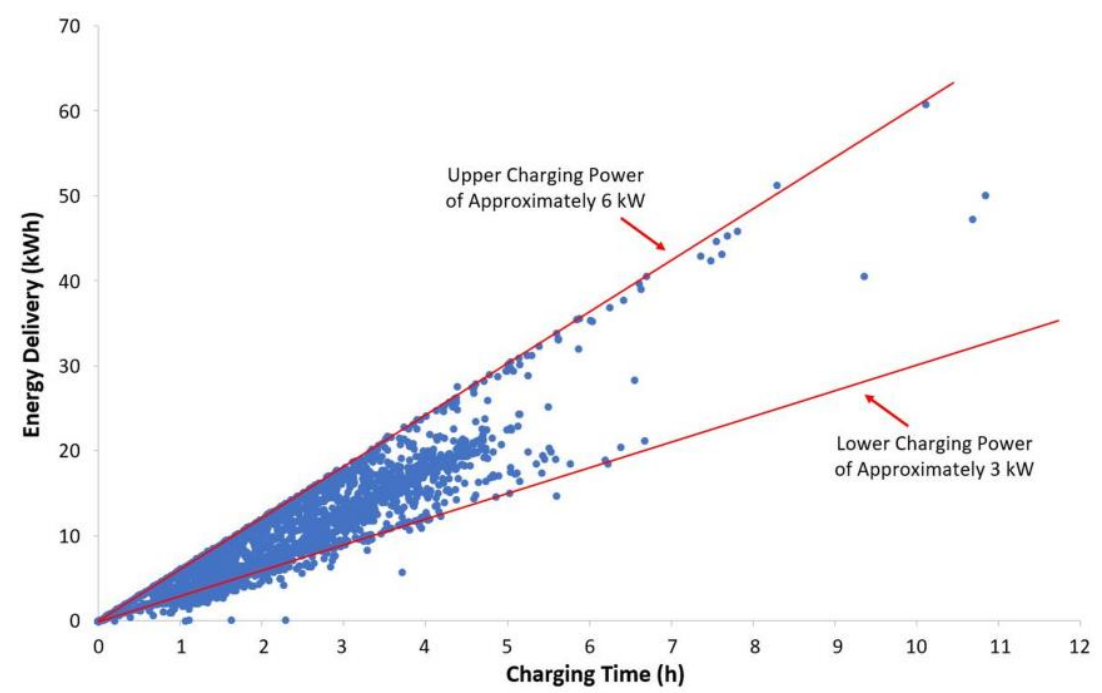

Figure 4. Energy delivery versus charging time for each charging event. 


\subsection{Occupancy}

Figure 5 indicates that $65.2 \%$ of charging events happened during typical UGA office hours (8:00 a.m. to 6:00 p.m., Monday through Friday). As shown in Figure 6, the number of charging events on Monday through Friday were very similar, ranging from 512 to 591, and the number of charging events on weekends were very similar, with 268 on Saturday and 229 on Sunday. Overall, $84.5 \%$ of charging events occurred on weekdays. The ratio of the average number of charging events on weekdays to the average number of charging events on weekends was 541.4 to 248.5 , or 2.2. Thus, the average daily usage on weekdays was more than double the average daily usage on weekends.

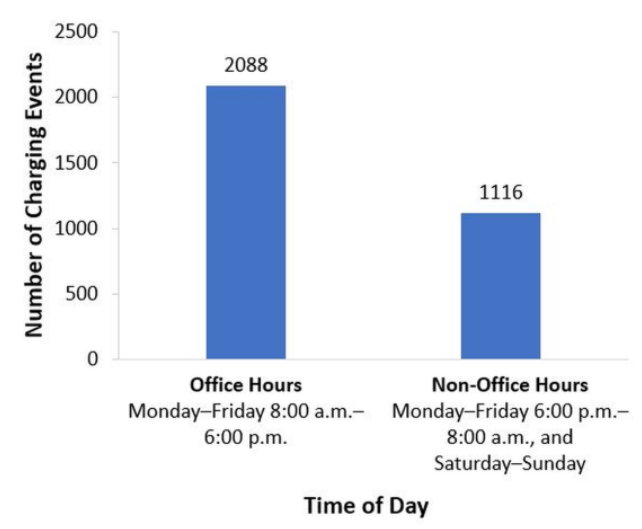

Figure 5. Office and non-office hour distribution.

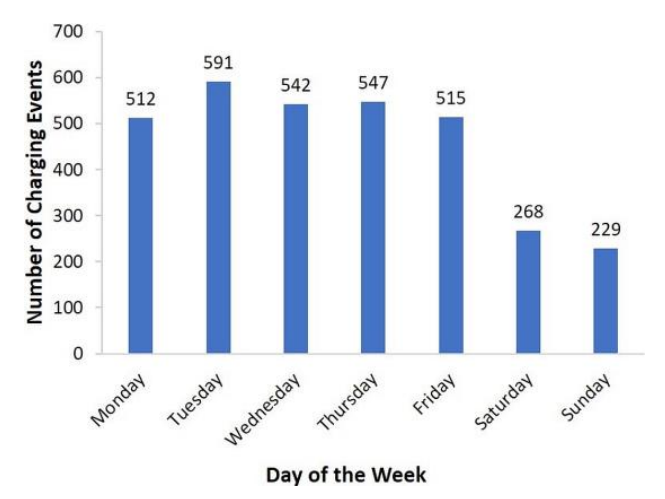

Figure 6. Distribution of charging events by day of the week.

As calculated from Figure 7, 58.2\% of charging events occurred between 7:00 a.m. and 2:00 p.m., and $35.3 \%$ of charging events occurred between 2:00 p.m. and 8:00 p.m. Therefore, $93.5 \%$ of charging events occurred between 7:00 a.m. and 8:00 p.m. The three most popular time periods for charging were (11:00 a.m., 12:00 p.m.], (12:00 p.m., 1:00 p.m.], and (5:00 p.m., 6:00 p.m.]. A possible explanation for these spikes is that the time ranges coincide with typical lunch and dinner hours. Other than these three periods, there were two noticeable trends, with similar numbers of charging events-in the low to mid two hundreds-in time periods from 7:00 a.m. to 2:00 p.m., and similar numbers of charging events—in the mid one hundreds—in time periods from 2:00 p.m. to 8:00 p.m. There was a fairly steady decrease in the number of charging events from 8:00 p.m. to 2:00 a.m., no charging events from 2:00 a.m. to 5:00 a.m., and a small number of charging events from 5:00 a.m. to 7:00 a.m.

The period of each bar in the histogram in Figure 8 is 65 days, and it can be seen that the frequency of charging events increased for the first six periods (390 days), from 51 to 302 charging events per period. The frequency then fluctuated for the next 12 periods, staying between 136 and 275 charging events. 
The onset of the electricity fee is indicated with an arrow in Figure 8, and it can be seen that the number of charging events increased for the next four periods ( 260 days) after the electricity fee was implemented. In Figure 9, it can be seen that charging station usage increased after the onset of the electricity fee.

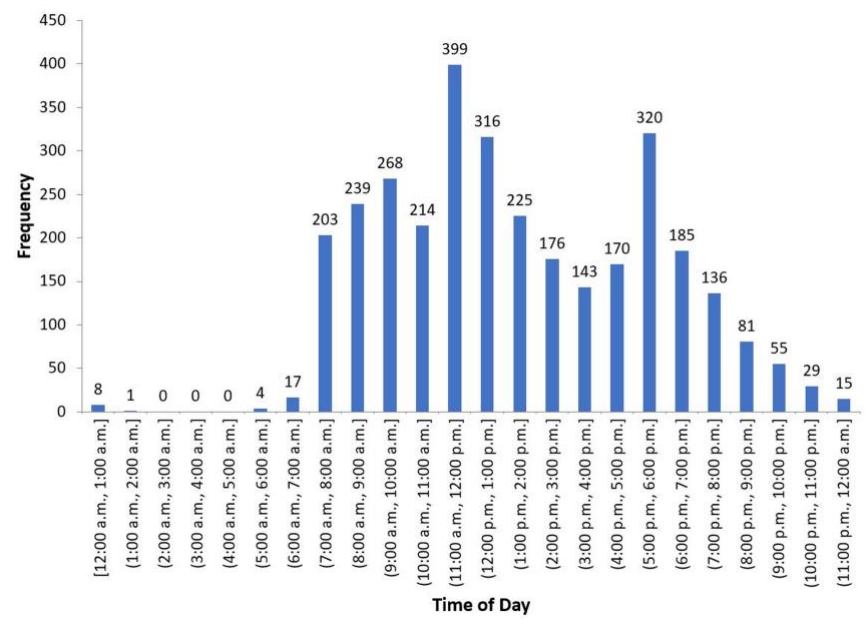

Figure 7. Histogram of charging events by time of day.

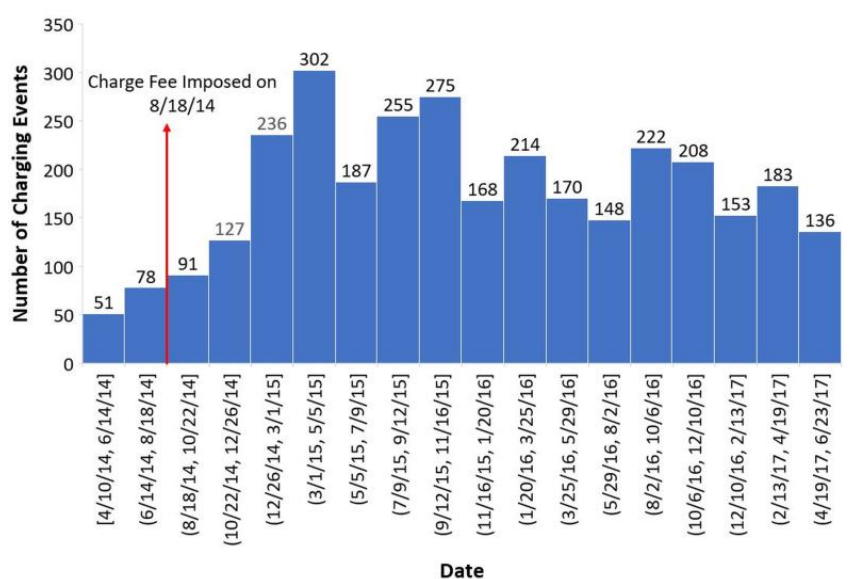

Figure 8. Distribution of charging events over time. Onset of electricity fee indicated with arrow.

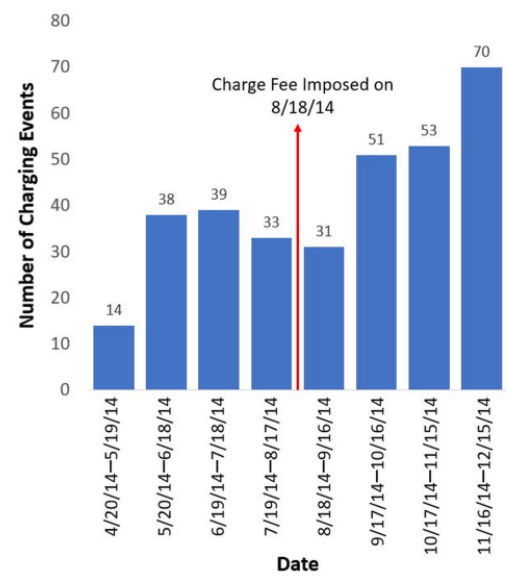

Figure 9. Frequency of charging events from four 30-day periods before and after electricity fee was implemented. Onset of electricity fee indicated with arrow. 


\subsection{Geolocation}

Only a few charging events were from out of state (Figure 10a), and the rest were from Georgia, mostly concentrated in Athens and the Greater Atlanta area (Figure 10b). Users from the Athens area comprised $49.41 \%$ of total charging events (Figure 10c). The most frequently occurring zip codes were 30605, 30606, 30607, and 30601, all of which are Athens zip codes. Overall, it appears that EVs were often used for short drives.

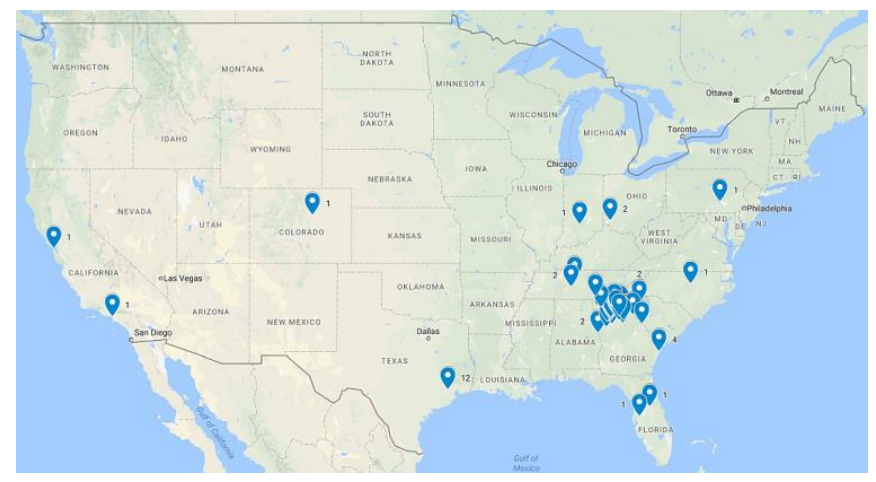

(a)

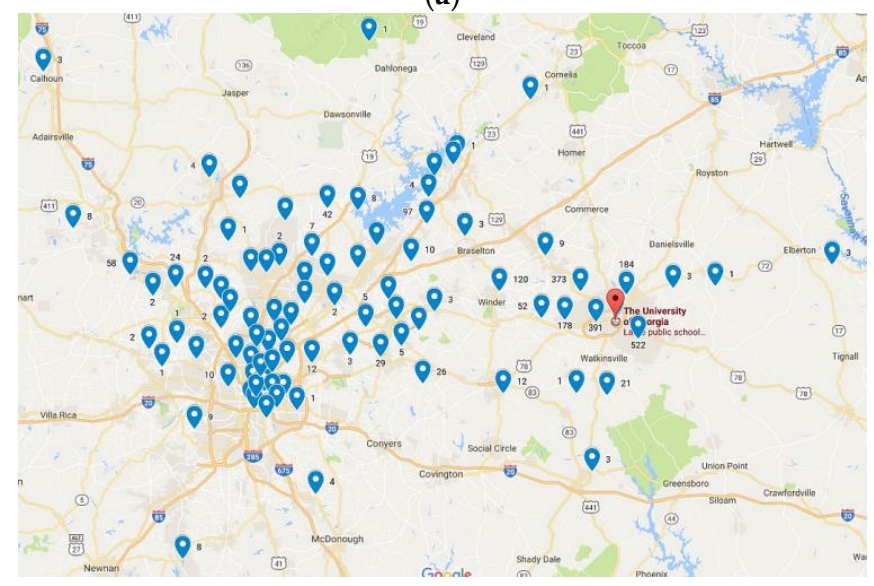

(b)

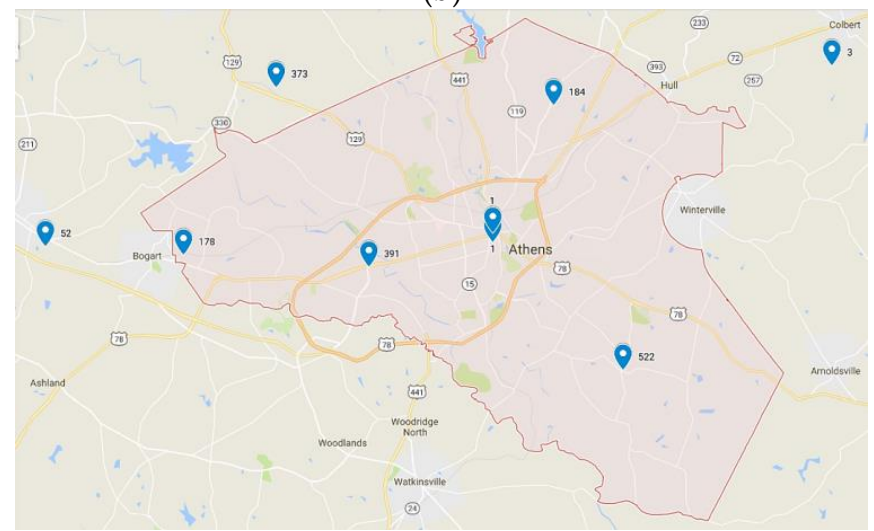

(c)

Figure 10. Zip codes associated with charging events. The number next to each marker indicates the frequency of that zip code: (a) Overall distribution including all valid zip codes; (b) Distribution of zip codes from the Athens and Greater Atlanta area. The red marker indicates the location of the University of Georgia; (c) Distribution of zip codes from Athens. 


\section{Discussion}

The methodology presented here focuses on providing a foundation of basic numerical and visual information about how and when charging stations are used.

The charging behavior section can be used to answer important questions about how charging stations perform. Different levels of charging stations have different specifications, so the energy and power delivery will differ accordingly. Moreover, it is expected that charging stations operate at a lower power on average than the standard power rating due to many factors, such as different EV batteries and initial battery levels. This section also shows how long users typically charge their EVs, as well as how long users remain parked. The combination of information about energy delivery and charging time provides a bigger picture about how charging stations are used.

Charging station occupancy also needs to be examined in relation to the time of day and the day of the week. The occupancy section explores how demand differs based on college office hours, day of the week, and hour of the day. It also shows how occupancy levels have changed over time, and how implementation of, or increases in, electricity fees have affected charging station occupancy.

Building additional charging stations on a college campus will affect EV drivers from many locations. If sustainability and promoting EV usage is of importance to decision-makers, the size of the area and the number of drivers impacted by charging stations is a key area of information needed for planning. The geolocation section seeks to provide a visual of the distribution of charging station users.

The information gathered using the proposed methodology is expected to assist decision making about EV charging infrastructure development on college campuses and the surrounding community. The methodology will be especially useful for other college towns that plan to build additional charging stations.

\subsection{Limitations}

Although the methodology can be applied to other universities, one limitation of the study is that the generalizability of the findings in this paper to other universities is unclear. Therefore, decision-makers should be cautious when using this paper as a reference for potential expectations in another university. Additionally, all charging stations at UGA are located in parking decks that require either a parking permit or, for visitors, payment according to hourly rates which vary by time of day and length of parking. This may complicate usage of the case study results if decision-makers are considering installing charging stations in parking areas other than parking decks, or in parking decks that have different permit costs or parking rates.

Another limitation to the study is the number of variables which can affect EV charging station location planning. For example, policies, incentives, and technical support can all affect how infrastructure is planned. However, this study focused on charging station performance. Moreover, this study considered only a few of the many possible performance indexes.

\subsection{Future Work}

Future work should focus on identifying which variables and performance indexes have the most practical applications in planning for building new EV charging stations on college campuses. For example, conducting EV driver behavioral analysis and categorizing charging point users into different clusters may yield important information.

\section{Materials and Methods}

Data was collected from three ChargePoint level 2 charging stations located at the University of Georgia in three parking decks, the North Campus Deck, East Campus Deck, and South Campus Deck [24]. Data was collected about each of 3204 charging events from 10 April 2014 to 20 June 2017.

ChargePoint collects many categories of information by default and sorts the information into columns in a spreadsheet. Five of the columns generated by ChargePoint were used in this study. 
These columns were entitled: "Start Date”, “Total Duration (hh:mm:ss)”, "Charging Time (hh:mm:ss)”, "Energy (kWh)", and "Driver Postal Code". These five columns were used to obtain nine categories of information. The start date and time of each charging event were grouped into the column called "Start Date" in the ChargePoint spreadsheet, so the first step was to separate the start date and time into two separate columns. Next, the start date was used to identify the day of the week of each charging event. The charging time was then subtracted from the total duration to find the non-charging parking time. Since total duration, charging time, and non-charging parking time are all lengths of time rather than times of day, the values were transformed from the default time format with units of (hh:mm:ss) to a decimal format with units of $(\mathrm{h})$. Energy $(\mathrm{kWh})$ delivered to an EV during a charging event is defined as charging power $(\mathrm{kW})$ multiplied by charging time $(\mathrm{h})$. To find the power delivery of each charging event, the energy was divided by the charging time.

The nine categories of data derived from the raw data are summarized in Table 1 . These datasets were used to determine information about the charging behavior, charging station occupancy, and geolocation of charging station users (Sections 2.1-2.3).

Table 1. Information obtained from raw data.

\begin{tabular}{|c|c|c|}
\hline Category & Definition & Units \\
\hline Start Day of Week & Day of week at start of charging event & unitless \\
\hline Start Time & Time at start of charging event & (hh:mm:ss a.m./p.m.) \\
\hline Total Duration & Total length of time for which EV is plugged into charging station & (h) \\
\hline Energy & Total amount of energy delivered to EV during charging event & $(\mathrm{kWh})$ \\
\hline Power & The power level during each charging event & $(\mathrm{kW})$ \\
\hline Driver Postal Code & $\begin{array}{l}\text { 5-digit zip code entered by the user during ChargePoint account } \\
\text { registration }\end{array}$ & unitless \\
\hline
\end{tabular}

\subsection{Charging Behavior}

The first aim was to extract information about the charging time, non-charging parking time, energy delivery, and power delivery of charging events. To accomplish this, the following graphs were created:

(1) Histogram of charging time (one hour per bar)

(2) Histogram of Non-Charging Parking Time (one hour per bar)

(3) Scatterplot of Energy vs. Start Date

(4) Scatterplot of Energy vs. Charging Time

The histogram of charging time displays the frequency of charging events which have a charging time in the range of $[0,1],(1,2],(2,3] \mathrm{h}$, etc. This shows the distribution of charging times across all charging events, and the graph can be used to easily see the most common lengths of charging time and calculate the percentage of charging times within any desired range, such as $[0,3] \mathrm{h}$. The histogram of non-charging parking time is used in the same way as the histogram of charging time, but it displays the length of time for which vehicles remained parked at the charging station after active charging was complete.

The scatterplot of energy vs. start date provides both a visual of the distribution of the amount of energy delivery across all charging events and the distribution of energy delivery over time.

The scatterplot of energy vs. charging time can be used to visualize the range of power levels observed during charging events. Two straight lines surrounding the majority of the points in the scatterplot can be drawn from the origin of the graph. Because energy divided by time is power, the slopes of the two lines are the upper and lower power levels observed at the charging stations. 
The charging stations used at UGA, which are ChargePoint level 2 chargers, can provide a maximum output power of $7.7 \mathrm{~kW}$ [25]. However, charging power varies subject to different EV batteries and initial battery levels. The scatterplot of energy vs. charging time can be used to compare the charging stations' maximum output power with the operational output power.

\subsection{Occupancy}

The second aim was to examine the occupancy of the charging stations at different times of day, days of week, and the distribution over time. To this end, the following graphs were created:

(1) Bar graph comparing the number of charging events which occurred during and outside of UGA office hours

(2) Bar graph comparing the number of charging events which occurred on each day of the week

(3) Histogram of frequency of charging events by time of day (one hour per bar)

(4) Histogram of frequency of charging events by date (65 days per bar)

(5) Bar graph comparing the number of charging events which occurred before and after implementation of an electricity fee

Typical UGA office hours are from 8:00 a.m. to 6:00 p.m. on Monday through Friday. To evaluate the effect of office hours on charging station occupancy levels, a bar graph was created to compare the number of charging events during and outside of typical office hours.

The distribution of charging station usage by day of the week is useful for determining if the charging stations are used evenly over the course of the week. A bar graph was created to compare the number of charging events which occurred on each day of the week. Of particular interest is whether the charging stations are used more, less, or the same on weekends compared to weekdays. Therefore, a ratio of the average number of charging events on weekdays to the average number of charging events on weekends was calculated.

The histogram of frequency of charging events by time of day shows the frequency of charging events which occurred at [12:00 a.m., 1:00 a.m.], (1:00 a.m., 2:00 a.m.], (2:00 a.m., 3:00 a.m.], etc. This graph can be used to calculate which individual hours of the day and which ranges of time are the most popular for charging. It can also be used to visualize the general pattern of occupancy observed over the course of a day.

To examine changes in charging station usage over time, a histogram of the frequency of charging events by date was created. The period of each bar in the histogram is 65 days, although this can be adjusted based on the amount of data collected so that overarching patterns can be observed rather than daily fluctuations.

Starting on 18 August 2014-the beginning of the fall semester-an electricity fee for charging was applied at a rate of $\$ 0.75 / \mathrm{h}$ for the first two hours, and then $\$ 1.5 / \mathrm{h}$ after that $[24,26]$. A bar graph was created comparing the number of charging events which occurred during each of four 30-day intervals before the onset of the electricity fee, and four 30-day intervals after the onset of the electricity fee at the charging stations (the first 30-day interval after the fee includes the first day the fee was implemented, which was 18 August 2014). This graph can be used to visualize and calculate whether the electricity fee coincides with any changes in charging station usage (especially whether occupancy decreases after fee implementation).

\subsection{Geolocation}

The third aim was to create a visual representation of the distribution of zip codes associated with each charging event. Drivers must enter their zip code during registration at the ChargePoint station. This data was collected for each charge event, although 225 charging events were associated with an invalid zip code. Using the zip codes of the remaining 2979 charging events, the following visual aids were created in Google Maps:

(1) Map showing distribution of all valid zip codes 
(2) Map showing distribution of zip codes from the Athens and Greater Atlanta area

(3) Map showing distribution of zip codes from Athens

In the maps, each marker represents one zip code, and the number next to each marker indicates the frequency of that zip code.

\section{Conclusions}

This study proposed a methodology to analyze EV charging station usage on a college campus, and the University of Georgia (UGA) in Athens, Georgia was used as a case study. Data was collected from three ChargePoint level 2 charging stations located in three parking decks at UGA. Information about 3204 charging events from 10 April 2014 to 20 June 2017 was recorded. The data was analyzed using the protocol presented to provide information about EV charging station usage at UGA in terms of charging behavior, charging station occupancy, and geolocation of charging station users.

The methods used in this study are reproducible for other cities, and the results will be useful for planning EV charging infrastructure development on other college campuses in the USA. For colleges that plan to build additional charging stations, the methodology presented can be used to examine past charging behavior at existing charging stations. The data collection method is time- and cost-effective because ChargePoint charging stations collect data about every charging event, and this data is quick, easy, and free to obtain. Additionally, the results from this case study can be used as a reference for possible expectations of EV charging station usage on other college campuses. This will be especially useful for colleges that do not have charging stations but want to introduce EV charging infrastructure to their campus.

Acknowledgments: This material is based upon work supported by the Georgia Research Academic Partnership in Engineering (GRAPE) program and the University of Georgia (UGA) Sustainability Grant. The authors would like to thank Jason Perry and Kevin Kirsche of the UGA Office of Sustainability for providing data for the study as well as their intellectual support. Additional thanks go to Michael Jerue of JuiceCar, LLC.

Conflicts of Interest: The authors declare no conflicts of interest.

\section{References}

1. EDTA. Electric Drive Sales Dashboard. Available online: http://electricdrive.org/index.php?ht=d/sp/i/ 20952/ pid/20952 (accessed on 11 October 2017).

2. FuelEconomy.gov. All-Electric Vehicles. Available online: http://www.fueleconomy.gov/feg/evtech.shtml\# endnotes (accessed on 11 October 2017).

3. FuelEconomy.gov. Federal Tax Credits for All-Electric and Plug-in Hybrid Vehicles. Available online: https: / /www.fueleconomy.gov / feg/taxevb.shtml (accessed on 11 October 2017).

4. Office of Energy Efficiency and Renewable Energy. Electric Vehicles: Tax Credits and Other Incentives. Available online: https:/ / energy.gov/eere/electricvehicles/electric-vehicles-tax-credits-and-other-incentives (accessed on 11 October 2017).

5. Hartman, K.; Dowd, E. State Efforts to Promote Hybrid and Electric Vehicles. Available online: http:/ / www. ncsl.org/research/energy/state-electric-vehicle-incentives-state-chart.aspx (accessed on 11 October 2017).

6. U.S. Energy Information Administration. Use of Energy in the United States Explained: Energy Use for Transportation. Available online: https:/ / www.eia.gov / energyexplained/?page=us_energy_transportation (accessed on 11 October 2017).

7. Francfort, J. Electric Vehicle Charging Levels and Requirements Overview. Available online: https:// avt.inl.gov/sites/default/files/pdf/presentations/CleanCitiesWedinarCharging12-15-10.pdf (accessed on 11 October 2017).

8. Chevrolet. Volt. Available online: http://www.chevrolet.com/electric/volt-plug-in-hybrid (accessed on 4 May 2018).

9. Schmidt, E. 2017 Battery Electric Cars Reported Range Comparison. Available online: https://www. fleetcarma.com/2017-battery-electric-cars-reported-range-comparison/ (accessed on 11 October 2017).

10. Chevrolet. Bolt ev. Available online: http:/ / www.chevrolet.com/bolt-ev-electric-vehicle (accessed on 4 May 2018). 
11. Tesla. Model 3. Available online: https://www.tesla.com/model3 (accessed on 4 May 2018).

12. Tesla. Model s. Available online: https://www.tesla.com/models (accessed on 4 May 2018).

13. Kameda, H.; Mukai, N. Optimization of charging station placement by using taxi probe data for on-demand electrical bus system. In Knowledge-Based and Intelligent Information and Engineering Systems; König, A., Dengel, A., Hinkelmann, K., Kise, K., Howlett, R.J., Jain, L.C., Eds.; Springer: Berlin/Heidelberg, Germany, 2011; Volume 6883, pp. 606-615.

14. Chen, T.D.; Kockelman, K.M.; Khan, M. The electric vehicle charging station location problem: A parking-based assignment method for seattle. In Proceedings of the 92nd Annual Meeting of the Transportation Research Board, Washington, DC, USA, 13-17 January 2013; pp. 28-36.

15. Ge, S.; Feng, L.; Liu, H. The planning of electric vehicle charging station based on grid partition method. In Proceedings of the 2011 International Conference on Electrical and Control Engineering (ICECE), Yichang, China, 16-18 September 2011; pp. 2726-2730.

16. Frade, I.; Ribeiro, A.; Gonçalves, G.; Antunes, A.P. Optimal location of charging stations for electric vehicles in a neighborhood in lisbon, portugal. Transp. Res. Rec. J. Transp. Res. Board 2011, 2252, 91-98. [CrossRef]

17. Oda, T.; Aziz, M.; Mitani, T.; Watanabe, Y.; Kashiwagi, T. Mitigation of congestion related to quick charging of electric vehicles based on waiting time and cost-benefit analyses: A japanese case study. Sustain. Cities Soc. 2018, 36, 99-106. [CrossRef]

18. Aziz, M.; Oda, T.; Ito, M. Battery-assisted charging system for simultaneous charging of electric vehicles. Energy 2016, 100, 82-90. [CrossRef]

19. Azadfar, E.; Sreeram, V.; Harries, D. The investigation of the major factors influencing plug-in electric vehicle driving patterns and charging behaviour. Renew. Sustain. Energy Rev. 2015, 42, 1065-1076. [CrossRef]

20. Moon, H.; Park, S.Y.; Jeong, C.; Lee, J. Forecasting electricity demand of electric vehicles by analyzing consumers' charging patterns. Transp. Res. Part D Transp. Environ. 2018, 62, 64-79. [CrossRef]

21. Athens-Clarke County Unified Government. Demographics. Available online: https://www.athensclarkecounty. com/105/Demographics (accessed on 11 October 2017).

22. Wikipedia. List of College Towns. Available online: https://en.wikipedia.org/wiki/List_of_college_towns\# College_towns_in_the_United_States (accessed on 11 October 2017).

23. Reviews, B.C. The 50 Best College Towns in America. Available online: https:/ /www.bestcollegereviews. org/50-best-college-towns-america/ (accessed on 11 October 2017).

24. Plugshare. Athens Public Charge Stations. Available online: https:/ /www.plugshare.com/ (accessed on 11 October 2017).

25. ChargePoint. General Chargepoint Home Faqs. Available online: https://www.chargepoint.com/products/ home/general/ (accessed on 11 October 2017).

26. Schupska, S. Uga Installs First Public Level 2 Electric Vehicle Charger in Athens Area. Available online: http: / / news.uga.edu/releases/article/first-public-level-2-electric-vehicle-charger-athens-area/ (accessed on 11 October 2017).

C 2018 by the authors. Licensee MDPI, Basel, Switzerland. This article is an open access article distributed under the terms and conditions of the Creative Commons Attribution (CC BY) license (http://creativecommons.org/licenses/by/4.0/). 